Résumés des conférences et travaux

\title{
Philologie italique
}

\section{Emmanuel Dupraz}

\section{(2) OpenEdition \\ Journals}

\section{Édition électronique}

URL : https://journals.openedition.org/ashp/3676

DOI : $10.4000 /$ ashp.3676

ISSN : 1969-6310

Éditeur

Publications de l'École Pratique des Hautes Études

\section{Édition imprimée}

Date de publication : 1 septembre 2020

Pagination : 116-118

ISSN : 0766-0677

\section{Référence électronique}

Emmanuel Dupraz, "Philologie italique », Annuaire de l'École pratique des hautes études (EPHE), Section des sciences historiques et philologiques [En ligne], 151 | 2020, mis en ligne le 09 juillet 2020, consulté le 06 juillet 2021. URL : http://journals.openedition.org/ashp/3676 ; DOI : https://doi.org/10.4000/ashp. 3676 


\title{
PHILOLOGIE ITALIQUE
}

\author{
Directeur d'études : M. Emmanuel DuPRAZ
}

Programme de l'année 2018-2019 : I. La notion de calendrier dans l'Italie d'époque républicaine (suite). - II. Comparatifs et superlatifs dans les langues sabelliques.

Les deux thèmes abordés en 2018-2019 ont été très différents. Le premier relevait d'une étude morphologique et sémantique de la catégorie des degrés de l'adjectif dans les langues sabelliques. Ont été étudiées toutes les occurrences d'équatif (exceptionnelles), de comparatif et de superlatif dont l'identification peut être considérée comme sûre et dont le contexte est assez bien documenté pour permettre une analyse qui s'est notamment attachée aux constructions dans lesquelles entrent ces formes de gradation.

L'examen de toutes ces occurrences a permis de reconstituer un système morphologique en principe simple : à des comparatifs en *ero- ou *-tero-correspondent des superlatifs en *-mo- ou *-tmmo-; à des comparatifs en *-is- (uel sim.) correspondent des superlatifs en *-is-mmo-.

La dénomination de comparatifs et superlatifs pour les formes de la série en *-ero- ou *-tero- resp. *-mo- ou *-tmmo- n'est pas entièrement justifiée : ces formes ne semblent pas pouvoir entrer dans des constructions avec compléments («plus X que », « le plus $\mathrm{X}$ de »). Cette série a une unité précise : les comparatifs et superlatifs concernés renvoient à un des deux termes extrêmes, ou à une des deux directions, sur une dimension qui prototypiquement est spatiale, à partir d'un centre déictique; ils fonctionnent donc par paires. Un point supplémentaire qui distingue des comparatifs et superlatifs au sens plein les formes concernées est qu'en général il n'existe pas de degré positif pour ces dernières, parce que les formes de comparatifs et de superlatifs en question sont dérivées d'adverbes qui semblent souvent archaïques et disparus de la langue en synchronie.

Néanmoins les formes de cette série partagent une propriété importante des comparatifs et des superlatifs au sens plein du terme : les formes en *-ero- ou *-tero-renvoient à une bipartition de la dimension spatiale concernée, telle que seuls deux termes extrêmes, ou deux directions, s'opposent l'une à l'autre; les formes en *-mo- ou *-tmmo-, au contraire, supposent apparemment la présence d'un ou plusieurs termes intermédiaires entre les deux termes extrêmes, du moins à en juger par les exemples très rares où il est possible d'évaluer le contexte et la référence des formes concernées (anglome. somo « vers l'angle suprême », s'agissant d'un templum augural, un rectangle à quatre angles).

La série des comparatifs en $*_{-i s-}$ (uel sim.) et des superlatifs en $*_{-i s-m o m o-\text {, en }}$ revanche, concerne des adjectifs qui désignent des propriétés quelconques, graduables, comme par exemple la «nouveauté » (nuvis, adverbe ombrien, « de manière plus neuve, postérieurement »; nuvime, adverbe ombrien, « de manière toute neuve, à la fin »; cf. lat. nouissimus « dernier »). 
Des difficultés apparaissent néanmoins, qui montrent que ce système ne rend pas pleinement compte du fonctionnement des comparatifs et des superlatifs sabelliques. Il existe des formes qui combinent les suffixes des deux systèmes. Une de ces formes, le superlatif ne/ssimas «le plus proche», ne paraît pas pouvoir être expliquée sans l'hypothèse que *-tmomo- et *-is-momo- ont été combinés à une date récente dans l'histoire de la langue. La préhistoire de ce superlatif, que son signifié spatial paraît assigner originellement au suffixe *-tmmo-, est complexe, d'autant qu'il existe des formes celtiques qui semblent nettement apparentées (par exemple gallois nesaf « le plus proche, prochain »).

L'étude de ce système morphologique a aussi permis des remarques de syntaxe, notamment sur le fonctionnement du comparatif nistrus « plus proche » et du superlatif ne/ssimas « le plus proche» : il apparaît que l'un et l'autre se construisent avec un complément à l'ablatif pour marquer le centre déictique (" plus proche de », « le plus proche de »), ce qui a des conséquences importantes pour l'interprétation sémantique et référentielle des inscriptions de Capoue dites iúvilas et de l'aménagement du sanctuaire dont elles proviennent.

L'autre thème abordé en 2018-2019, dans la suite d'une étude entreprise les années précédentes, a été celui des calendriers préromains. C'est un document celtique, le calendrier gaulois sur bronze de Coligny, qui a été analysé. Ce document $\mathrm{du} \mathrm{II}^{\mathrm{e}}$ siècle de notre ère renvoie à un calendrier luni-solaire particulièrement élaboré, qui atteste une réflexion poussée sur les cycles lunaire et solaire et aussi sur la valence théologique et rituelle de ceux-ci. Le séminaire s'est attaché en particulier à souligner les oppositions binaires, entre années, entre semestres, entre mois, entre quinzaines du mois, entre jours, et même semble-t-il entre subdivisions du jour, que ce calendrier permet de reconstituer (avec une certitude variable il est vrai). Le calendrier de Coligny partage avec celui de la Rome républicaine ce principe d'oppositions binaires, mais d'une manière qui ne suppose pas nettement une influence directe de Rome sur les Gaulois de Coligny et qui renvoie plutôt à une réflexion très originale sur la base des cycles naturels, à partir de la notion de bipartition, commune aux deux cultures. Ainsi, à Rome, le contraste entre la nouvelle lune et la pleine lune est interprété en termes de contraste entre les sexes (entre Junon, souveraine de la nouvelle lune, et Jupiter, souverain de la pleine lune) ; à Coligny, le principe formel de bipartition apparaît notamment dans l'opposition entre mois « bons » et mois « non-bons », elle-même pertinente, par exemple, pour définir un semestre majoritairement « bon » et un autre majoritairement «non-bon ». Il est regrettable que la concision du document interdise de connaître les conséquences de ces oppositions complexes emboîtées, sur les rituels et même sur la vie courante.

Le séminaire a accueilli en avril 2019 M. Carlos Jordán Cólera, Profesor à l'université de Zaragoza (Espagne), qui a donné, dans les quatre séances qui lui étaient imparties, une introduction très complète et très riche (elle sera bientôt publiée en français) à la langue celtibère, c'est-à-dire à celle des langues indo-européennes de l'Ibérie ancienne qui est la mieux connue. Mettant en évidence autant les différents genres épigraphiques de textes qui sont documentés que les catégories morphologiques qui apparaissent dans chacun d'entre eux, M. Carlos Jordán Cólera a permis 
à l'auditoire francophone du séminaire d'avoir accès aux progrès énormes accomplis dans l'analyse du corpus celtibère par nos collègues espagnols dans les dernières décennies. Les problématiques communes aux chercheurs qui travaillent sur les différentes langues d'attestation fragmentaire du bassin méditerranéen antique justifient pleinement la prise en compte du très riche corpus de langues et de documents que fournit la péninsule Ibérique, à côté de l'Italie et dans une moindre mesure de la Gaule et de l'Afrique du Nord anciennes. 